\title{
220 TRANSFECTION OF HUMAN ADIPOSE-DERIVED MAST CELLS: A CHARACTERISTIC STUDY USING DIFFERENT CHEMICAL REAGENTS
}

${ }^{1}$ Mona Motaghed*, ${ }^{2}$ Christopher Kepley, ${ }^{1}$ Kristen Dellinger, ${ }^{2}$ Mohammad Fereydouni, ${ }^{1}$ Elnaz Ahani. ${ }^{1}$ North Carolina AandT State University, Greensboro, NC, USA; ${ }^{2}$ The University of North Carolina at Greensboro (UNCG), Greensboro, NC, USA

Background Mast cells are essential initiators and regulators in both innate and adaptive immunity in addition to being mediators of Type I hypersensitivity. Their role in cancer pathogenesis has been challenging to assess, given they contain both pro- and anti-tumorigenic mediators. Thus, to elucidate the functions of mast cell-derived gene products in cancer pathogenesis and mast cell differentiation, it is critical to study them by comparing their functional responses between those with selected gene knockdown and those without. However, primary human mast cells are known as hard-to-transfect cells and a fast and efficient method for transient transfection of primary human adipose mast cells has not been reported.

Methods We investigated different approaches to transfect primary human adipose-derived mast cells from 10 donors (24 55 yrs.). Three cationic lipid nanoparticles (Metafectene and Metafectene-Pro, Biontex Laboratories, and Lipofectamine 3000, Thermo Fisher), one lipid/histone-based nanoparticle (Turbofectin 8.0, OriGene), and two non-liposomal polymeric reagents (TransIT-LT1 and TransIT-2020, MirusBio) were evaluated in conjunction with green fluorescent protein (GFP)tagged genes (e.g., tryptase) at different ratios of DNA plasmid to the reagent. The functional and phenotypic responses of the mast cells were compared to non-transfected mast cells. Results We found that transfection of primary human adipose mast cells is highly donor-dependent. Parameters that affect transfection efficiency include donor age, stem cell isolation procedure, in vitro age of mast cells at the time of transfection, the type and dosage of the transfection reagent, incubation time, presence of antibiotics, and serum content. The transfection rate efficiencies of human mast cells using lipid synthetic nanoparticles was higher than the lipid/histone-based and non-liposomal polymeric reagents. The maximum Median Fluorescence Intensity (MFI) fold change compared to the non-transfected mast cells was about 17 for the youngest donor that was transfected 7 to 10 weeks after isolation using Metafectene-Pro.

Conclusions In general, older donor mast cells and mast cell progenitors were less susceptible to transfection. Also, a higher transfection rate is accompanied by increased cytotoxicity, so the analysis of various transfection conditions limiting cell death was the primary goal. Metafectene-Pro significantly increased GFP reporter gene expression compared to the other reagents. The transfected mast cells were phenotypically and functionally similar to non-transfected cells and were viable compared to control mast cells from the same donors. These studies will aid in determining if mast cell mediators have pro- or anti-tumor cell effects to help define the role these cells have in cancer pathogenesis.

Ethics Approval This study was approved by UNCG IRB, approval number 12-099, and under the NIH-defined "exempt" status for human subjects research.

http://dx.doi.org/10.1136/jitc-2021-SITC2021.220 